
\title{
3 Research Square \\ The pH Effects on SARS-CoV and SARS-CoV-2 Spike Proteins in the Process of Binding to hACE2
}

\section{Yixin Xie}

The University of Texas at El Paso

\section{Wenhan Guo}

The University of Texas at El Paso

\section{Alan Lopez-Hernadez}

The University of Texas at El Paso

\section{Shaolei Teng}

Howard University

Lin Li ( $\square$ lli5@utep.edu )

The University of Texas at El Paso

\section{Research Article}

Keywords: COVID-19, electrostatic features, SARS-CoV/SARS-CoV-2, hACE2

Posted Date: September 9th, 2021

DOl: https://doi.org/10.21203/rs.3.rs-871118/v1

License: (c) (1) This work is licensed under a Creative Commons Attribution 4.0 International License.

Read Full License

Version of Record: A version of this preprint was published at Pathogens on February 11th, 2022. See the published version at https://doi.org/10.3390/pathogens11020238. 


\section{The pH Effects on SARS-CoV and SARS-CoV-2 Spike Proteins in the Process of Binding to hACE2}

$6 \quad{ }^{1}$ Computational Science Program, University of Texas at El Paso, El Paso, TX.

$7 \quad 2$ Department of Biology, Howard University, Washington, D.C.

$8 \quad{ }^{3}$ Department of Physics, University of Texas at El Paso, El Paso, TX.

10 *Correspondence:

11 Lin Li: 1li5@utep.edu

Keywords: SARS-CoV, SARS-CoV-2, COVID-19, Electrostatic Features, AngiotensinConverting Enzyme 2, hACE2, spike protein, $\mathrm{pH}$ dependence, binding energy, folding energy

\section{Abstract}

COVID-19 has been threatening human health since the late 2019, which has significant impact on human health and economy. Understanding the SARS-CoV-2 and other coronaviruses is important to develop effective treatments for COVID-19 and other coronaviruses-caused diseases. In this work, we applied multi-scale computational approaches to study the electrostatic features of spike (S) proteins for SARS-CoV and SARS-CoV-2. From our results, we found that SARS-CoV and SARS-CoV-2 have similar charge distributions and electrostatic features when binding with the human angiotensin-converting enzyme 2 (hACE2). The energy pH-dependence calculations revealed that the complex structures of hACE2 and the S proteins of SARSCoV/SARS-CoV-2 are stable at $\mathrm{pH}$ values ranging from 7.5 to 9. Molecular dynamics simulations were performed using NAMD to investigate the hydrogen bonds between $\mathrm{S}$ proteins and hACE2. From the MD simulations it was found that SARS-CoV-2 has four pairs of essential hydrogen bonds (high occupancy, >80\%), while SARS-CoV has three pairs, which indicates the SARS-CoV-2 S protein has relatively more robust binding strategy than SARS-CoV S protein.

31 Four key residues forming essential hydrogen bonds from SARS-CoV-2 are identified, which are potential drug targets for COVID-19 treatments. The findings in this study shed lights on the

33 current and future treatments for COVID-19 and other coronaviruses-caused diseases. 


\section{Introduction}

36 The ongoing COVID-19 pandemic is changing human society significantly and causing both economic and social consequences all over the world [1]. Coronaviruses are named for their crown-like spikes on their surface, and they are commonly found in many mammal species [2]. Human coronaviruses were firstly identified in the mid-1960s. There are four main subgroupings of coronaviruses, known as alpha, beta, gamma, and delta [3]. Among all the coronaviruses, there are seven known types of coronaviruses that can infect human beings. People around the world commonly get infected by human coronaviruses 229E, NL63, OC43, and HKU1 $[4,5]$. And some coronaviruses that infect animals are able to evolve and infect humans, among which the three recent cases are SARS-CoV-2, SARS-CoV, and MERS-CoV[6]. The SARS-CoV-2 virus is the novel coronavirus that causes coronavirus disease 2019, or COVID-19. Other than COVID-19, coronaviruses have caused several pandemics before, including severe acute respiratory syndrome (SARS) which was caused by SARS-CoV and the Middle East respiratory syndrome (MERS) which was caused by MERS-CoV. To end the current pandemic soon and be prepared for the future similar challenges for human society, it is essential to understand the binding mechanisms of SARS-CoV-2 infecting human cells. This is achievable by studying the stabilities of SARS-CoV-2 at different $\mathrm{pH}$ conditions, and identify the key residues that play significant roles in the binding processes.

Coronaviruses contain membrane glycoprotein $(\mathrm{M})$, nucleocapsid protein $(\mathrm{N})$, spike protein $(\mathrm{S})$, envelope protein (E) and an RNA single chain[7]. For all enveloped viruses, one of the most important steps during the binding process is membrane fusion, which allows viruses to get into host cells [8]. For coronaviruses, the fusion protein is the $S$ protein that leads the binding process to attack human cells through the host cell receptor angiotensin-converting enzyme 2 (hACE2) [9]. Human hACE2 (hACE2) is an enzyme located widely in the human body, including the lungs, kidneys, adipose tissue, central nervous system and cardiovascular system [9-11] and it has multiple essential functions such as the regulation of amino acid transport in the kidney controlling the blood pressure, and viral receptors including both SARS-CoV-2 and SARS-CoV [11]. Since it is of extreme importance to human health, there are numerous research groups have been or are currently working on S proteins and hACE2 using various approaches.

The traditional process of the de novo drug design is a challenging task which consumes resources and time significantly. With the fast developments of computing technology, computational methods have been widely used to drug-related research[12], including proteinprotein interactions [13, 14], MD simulations[15], coarse-grained models[16], pH dependence of protein-protein interactions[17-20], etc. Our previous studies have applied multi-scale computational methods to study several pathogens [21-25] including the SARS-CoV-2 viruses $[26,27]$, which revealed some mechanisms of the SARS-CoV-2 S protein. Besides, many other research groups have made successful progress to understand the SARS-CoV-2 using computational methods [28, 29]. 
In this work, we first calculated the electrostatic potentials on the surface of S proteins from both SARS-CoV and SARS-CoV-2, followed by the electric field line comparison between SARS$\mathrm{CoV}$ and SARS-CoV-2 when they bind to hACE2. We found that the two viruses have similar $\mathrm{pH}$ responses: The $\mathrm{pH}$-dependence of folding energies for $\mathrm{S}$ protein receptor binding domains (RBDs) demonstrated that both the $\mathrm{S}$ protein RBDs of these two viruses are at the most stable status when $\mathrm{pH}$ values ranging from 6 to 9. Also, the $\mathrm{pH}$-dependence of binding energies for $\mathrm{S}$ protein RBDs and hACE2 RBD showed that the complex structures of the two viruses are at the most stable status at $\mathrm{pH}$ values ranging from 7.5 to 10.5. Therefore, SARS-CoV and SARS-CoV2 survive in a similar $\mathrm{pH}$ environment. The $\mathrm{pH} 7.5$ to 9 is the best condition for both SARS-CoV and SARS-CoV-2 to best perform their functions to bind with hACE2. Also, we analyzed the trajectories from 100ns MD simulations using NAMD [30] and identified hydrogen bonds with the involved key residues using VMD [31]. It is shown that for the high-frequency $(>80 \%)$ hydrogen bonds, SARS-CoV-2 has four pairs while SARS-CoV has three pairs, which indicates that the S protein of SARS-CoV-2 uses more residues to form strong hydrogen bonds. The key residues forming essential hydrogen bonds from SARS-CoV-2 are ARG-121, TYR103, THR182 and TYR171, which are potential drug targets for COVID-19 treatments. Using multiple computational approaches, the findings in this work pave the way for the current and future treatment development of COVID-19 and other coronaviruses-caused diseases.

\section{Methods}

\subsection{Structure Preparation}

The complex structures of SARS-CoV/hACE2 and SARS-CoV-2/hACE2 were downloaded from the Protein Data Bank (PDB ID 6ACG [32] and 7AD1 [33], respectively) . Please note that in 7AD1, the mutations that the authors made during their experiments are not on the interface area. Since we only focus on the interface area between S proteins and hACE2, the mutations do not affect our results. In this work, we used the complex structures to study the electrostatic binding interactions and the relative binding energies in different $\mathrm{pH}$ environments between $\mathrm{S}$ proteins and hACE2 RBDs. For the missing loops in proteins, we used MODELLER [34] to model the structures based on the sequences. To understand the mechanisms of $\mathrm{S}$ protein binding to hACE2 at the interface particularly, S protein RBDs were separated from the hACE2 binding

\subsection{Electrostatic Potential Calculation}

109 In order to study the electrostatic features, DelPhi [35, 36] was utilized to calculate the 110 electrostatic potential for the S proteins and hACE2 RBDs. In the framework of continuum 111 electrostatics, DelPhi calculates the electrostatic potential $\phi$ (in systems comprised of biological 
112 macromolecules and water in the presence of mobile ions) by solving the Poisson-Boltzmann

113 equation $(\mathrm{PBE})$ :

$$
\nabla \cdot[\epsilon(r) \nabla \phi(r)]=-4 \pi \rho(r)+\epsilon(r) \kappa^{2}(r) \sinh \left(\phi(r) / k_{B} T\right)
$$

115 where $\phi(r)$ is the electrostatic potential, $\epsilon(r)$ is the dielectric distribution, $\rho(r)$ is the charge 116 density based on the atomic structures, $\kappa$ is the Debye-Huckel parameter, $\mathrm{k}_{\mathrm{B}}$ is the Boltzmann

117 constant, and T is the temperature. Due to the irregular shape of macromolecules, DelPhi uses a 118 finite difference (FD) method to solve the PBE.

Before the DelPhi calculations, the PQR file of each trimer was generated by PDB2PQR [37]. We used AMBER [38]force field for PDB2PQR calculation, and removed water molecules. For the better results, we ensured the new atoms are not rebuilt too close to existing atoms and optimized the hydrogen bonding network.

During DelPhi calculations, the resolution was set as 0.5 grids/A. The dielectric constants were set as 2.0 for protein and 80.0 for the water environment, respectively. The $\mathrm{pH}$ value for the solvent environment was set to be 7.0. The probe radius for generating the molecular surface was 1.4 ̊. Salt concentration was set as $0.15 \mathrm{M}$. The boundary condition for the Poisson Boltzmann equation was set as a dipolar boundary condition. The calculated electrostatic potential on the surface was visualized with Chimera (figure 2). VMD was used to illustrate electric field lines between S protein and hACE2 (figure 3). Finally, the color scale range was set to be from -1.0 to $1.0 \mathrm{kT} / \mathrm{e}$ for the best visual presentation. Besides the calculations of electrostatic potentials, we also used DelphiForce [39] to calculate the electrostatic binding forces between each $S$ protein and hACE2 while separating them in the direction of the mass center connection line (figure S2). Besides the net forces between each S protein and hACE2, the X, Y, Z components of the net forces are also calculated and shown in figure $\mathrm{S} 2$.

\subsection{Relative Folding Energy Calculation}

139 We used DelPhiPKa $[40,41]$ to calculate $\mathrm{pKa}$ values of DNA and UDG, given the $\mathrm{pH}$ ranging from 0 to 14 with the $\mathrm{pH}$ interval of 0.5. During the calculations, we used AMBER force field, and removed water molecules and HETATM. For the hydrogen of ASP and GLU attached atom,

142 we used OD1 and OE1, respectively. Variance of Gaussian Distribution was set to be 0.7, salt

143 concentration was 0.15 , reference dielectric was 8.0, and external dielectric was 80.0.

145 The net charges of proteins at the unfolded state were calculated using this equation:

$$
Q_{u}(p H)=\sum_{i=1}^{N} \frac{10^{-2.3 y(i)(p H-p K a(i))}}{1+10^{-2.3 y(i)(p H-p K a(i))}}
$$


where the summation is of all the titratable groups, $y(i)$ value is -1 for acidic groups and +1 for basic groups, respectively. As for the folding free energy, we used this equation:

$$
\Delta \mathbf{N}\left(\mathbf{p} H_{\text {folding }}\right)=2.3 \mathrm{RT} \int_{p H_{i}}^{p H_{f}}\left(Q_{f}(p H)-Q_{u}(p H) d(p H)\right)
$$

where $Q_{f}(p H)$ and $Q_{u}(p H)$ stand for the net charge of folded and unfolded state, respectively. $\mathrm{R}$ is the universal gas constant taken as $1.9872 \times 10^{-3} \frac{\mathrm{kcal}}{\mathrm{Mol} * \mathrm{~K}}$. $\mathrm{T}$ is the temperature with the value of $300 \mathrm{~K}$.

Please note that the algorithms we applied to calculate the folding energies are for the relative values, that is, at $\mathrm{pH}=0$ the folding energy is 0 and at any other $\mathrm{pH}$ values the folding energies are the relative values to the $\mathrm{pH}=0$ condition.

\subsection{Relative Binding Energy Calculation}

For the binding energy calculation, we involved two methods, which are DelPhiPKa and MM/PBSA[42]. To calculate binding energy using DelPhiPKa, the following equation was used:

$$
\Delta N\left(p H_{\text {binding }}\right)=2.3 R T \int_{p H_{i}}^{p H_{f}}\left(Q_{t}(p H)-Q_{n}(p H)-Q_{r}(p H)\right) d(p H)
$$

where $\Delta N\left(p H_{\text {binding }}\right)$ is the the binding free energy at different $\mathrm{pH}$ values, $Q_{t}(p H), Q_{n}(p H)$, and $Q_{r}(p H)$ are the net charges of complexes of each model. $\mathrm{R}$ is the universal gas constant taken as $1.9872 \times 10^{-3} \frac{\mathrm{kcal}}{\mathrm{Mol} * \mathrm{~K}}$. $\mathrm{T}$ is the temperature with the value of $300 \mathrm{~K}$.

Please note that the algorithms we applied to calculate the binding energies are for the relative values, that is, at $\mathrm{pH}=0$ the binding energy is 0 and at any other $\mathrm{pH}$ values the binding energies are the relative values to the $\mathrm{pH}=0$ condition.

\subsection{Molecular Dynamic (MD) Simulations}

To simulate the dynamic interactions between $\mathrm{S}$ proteins RBD and hACE2 protein, MD simulations [15] were carried out using NAMD [30] with the help of GPUs on Lonestar5 clusters at the Texas Advanced Computing Center (TACC https://www.tacc.utexas.edu/). A 2000-step minimization was performed for each simulation, followed by a 100 million steps, during which 20,000 frames were saved from two 100ns simulations of both SARS-CoV and SARS-CoV-2 separately (1.0 fs per step, 1 frame at each 5000 steps, 100 million steps in total). The RMSDs of the SARS-CoV and SARS-CoV-2 trajectories are about 3.4 $\AA$ and $1.1 \AA$, respectively (figure S1). During the MD simulations, we used CHARMM [43] force field, the temperature was set to be 
$178300 \mathrm{~K}$, and the pressure was set to be standard using the Langevin dynamics. For PME, which is

179 set for full-system periodic electrostatics, with the grid size $(86,88,132)$ as $(\mathrm{x}, \mathrm{y}, \mathrm{z})$ value

180 respectively. In those two simulations, atoms that are not located in binding domains were

181 constrained within a margin of $10.0 \AA$ of their natural movement maximum length values. In

182 order to get a more accurate result of the simulation, data of the last $50 \mathrm{~ns}$ of simulations were

183 selected and used for data analysis, since the structure of the first $50 \mathrm{~ns}$ is not as stable as the last

$18450 \mathrm{~ns}$ of simulations. The simulation processes are visualized in movies 4 and 5 , generated by

185 VMD.

186

187 To analyze the interaction between $\mathrm{S}$ proteins and hACE2, the hydrogen bonds that formed

188 within the distance of $4 \AA$ were extracted from the last 10,000 frames (50 ns) of simulations. The

189 several top-strongest hydrogen bonds in each binding domain were determined by calculating

190 their formation frequency (the frequency in figure of essential hydrogen bonds is shown in figure 1917.

192

\section{Results and Discussions}

194 First of all, the electrostatic features of SARS-CoV and SARS-CoV-2 S proteins were

195 investigated, including electrostatic potential and electric field lines. Secondly, the relative

196 binding energies of complex structures and folding energies of S proteins at different $\mathrm{pH}$ values

197 were analyzed. Finally, the hydrogen bonds and related key residues in each complex structure

198 were obtained using MD simulations.

199

200

\subsection{S Protein Trimer Structure}




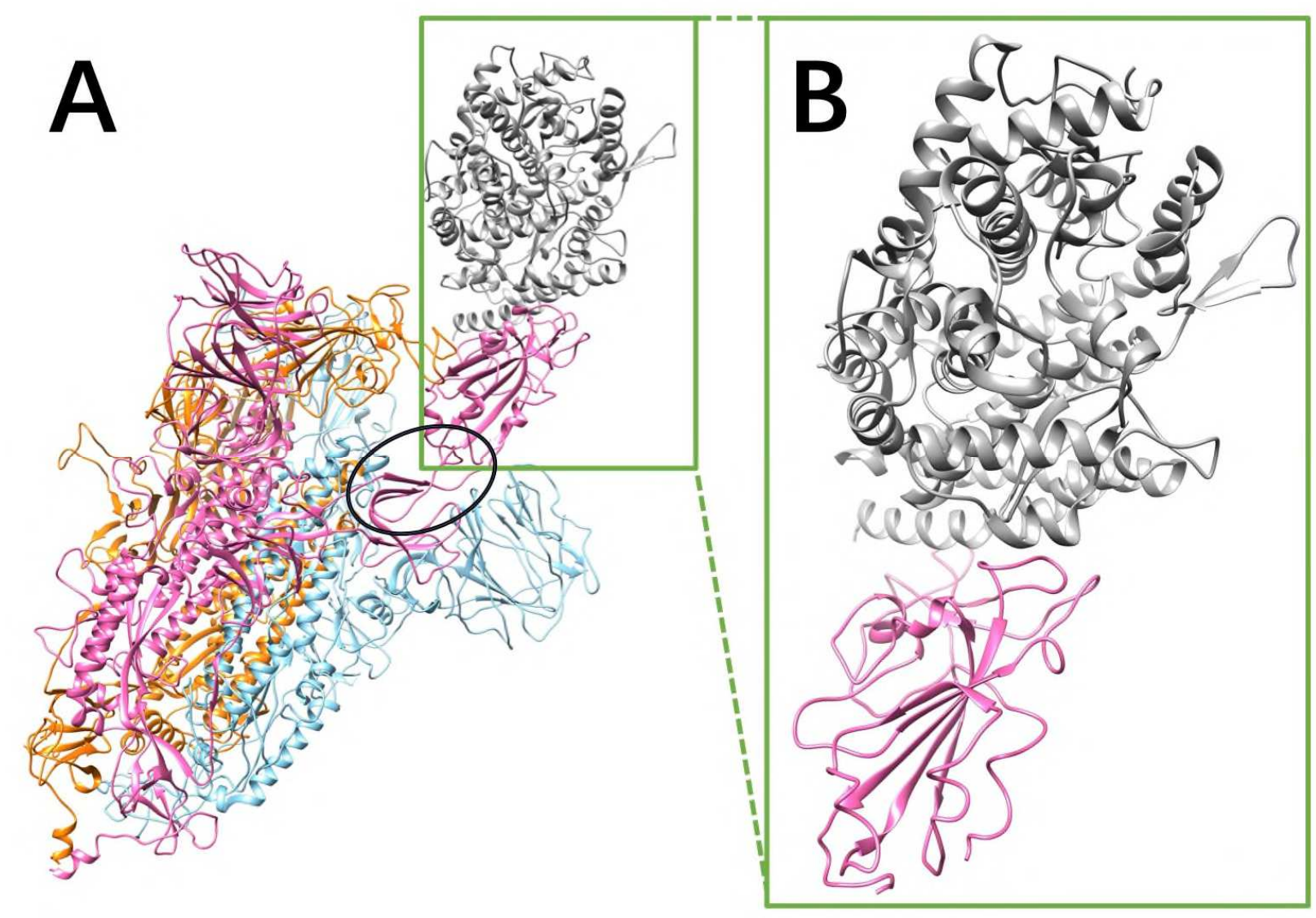

Figure 1. SARS-CoV S protein structure. Only the SARS-CoV S protein structure is illustrated in this figure, because SARS-CoV and SARS-CoV-2 S proteins are very similar (the RMSD between two S protein RBDs is $0.973 \AA$ ). (A) The $\mathrm{S}$ protein is a homotrimer (orange, blue, pink), of which one chain (pink) flips out when it binds to hACE2 (gray). The hinge connecting the $\mathrm{RBD}$ and the other part of $\mathrm{S}$ protein is shown in a black circle; (B) The closeup view of binding domains when S protein RBD (pink) binds to hACE2 RBD (gray).

211 The RMSD between the S proteins of SARS-CoV and SARS-CoV-2 is $0.973 \AA$, showing that 212 the S proteins of SARS-CoV and SARS-CoV-2 are very similar. The S proteins of SARS-CoV 213 and SARS-CoV-2 are both homotrimers. Each monomer contains an RBD which connects the 214 other part of the monomer via a hinge composed by two flexible loops (as shown in the black 215 circle of figure 1A). The RBD is in closed configuration when there is no hACE2 binds to the $\mathrm{S}$ 216 protein. When binding to hACE2, the RBD of one monomer flips out as open configuration and 217 it binds to the RBD of hACE2.

\subsection{Electrostatic Potential on Surfaces}


To study the electrostatic features, DelPhi was utilized to calculate the electrostatic potential on surfaces of the S protein trimer (full structure) and hACE2 RBD. The electrostatic potential distribution on SARS-CoV S protein trimer structure is showed in figure $2 \mathrm{BEH}$ and movie 1 , which were rendered by Chimera with a color scale from -1.0 to $1.0 \mathrm{kT} / \mathrm{e}$. The charge distribution on SARS-CoV-2 S protein trimer structure is shown in figure 2CFI and movie 2, which were rendered by Chimera with a color scale from -1.0 to $1.0 \mathrm{kT} / \mathrm{e}$ as well, for the comparison. Negatively and positively charged areas are colored in red and blue, respectively.

By comparing the electrostatic potential on surfaces of two trimer structures, it is obvious that the charge distribution of SARS-CoV and SARS-CoV-2 S proteins are different. From the top view (figure $2 \mathrm{~A}-\mathrm{C}$ ) and the bottom view (figure $2 \mathrm{G}-\mathrm{I}$ ), we noticed that SARS-CoV has slightly more positively charged area (blue), compared to SARS-CoV-2. It indicates that the SARS-CoV may attract the hACE2 more easily, since the hACE2 binding interface is overall negatively charged (movie 3). Such finding supports the previous studies of our research group [26, 27]. The electrostatic distribution differences observed from front views (figure 2D-F) of the $\mathrm{S}$ proteins demonstrate that the electrostatic features may have impacts on the stabilities of the trimers. Here it was not investigated several details about the binding stabilities among monomers in an S protein, due to the scope of this work that mainly focusses on the binding between S protein and hACE2. The electrostatic distributions on S protein RBDs show that the SARS-CoV RBD is more positive, which is consistent with the top view (figure 2BC). The bottom of the SARS-CoV (Figure 2EH) has more positive potential than SARS-CoV-2 (figure 241 2FI). 


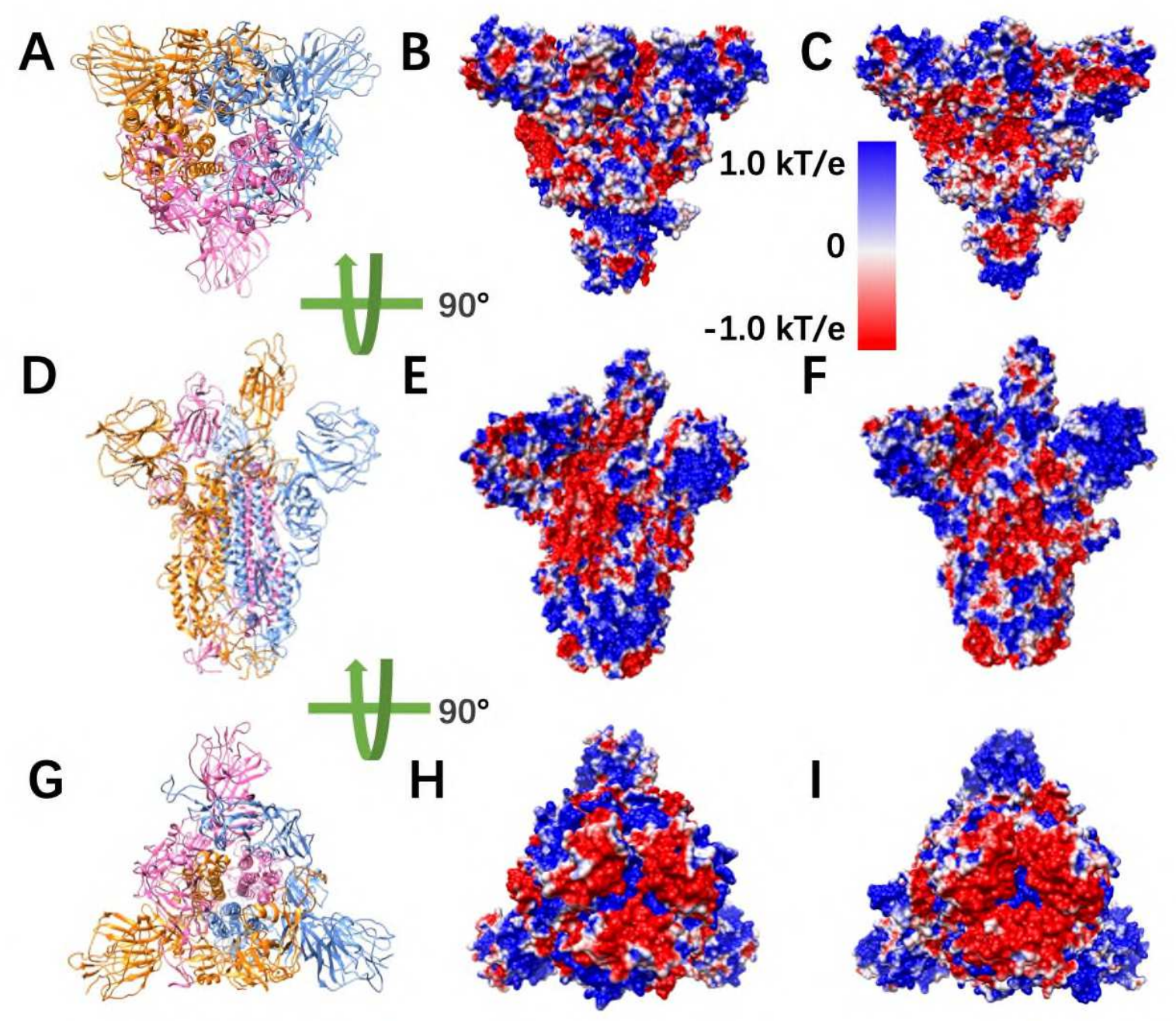

244 Figure 2. Electrostatic potential on surfaces of SARS-CoV and SARS-CoV-2 S proteins. (A)

245 Top view of S protein structure; (B-C) Top views of electrostatic potential on surfaces of SARS-

$246 \mathrm{CoV}$ and SARS-CoV-2 S protein, respectively; (D) Front view of S protein structure; (E-F)

247 Front views of electrostatic potential on surfaces of SARS-CoV and SARS-CoV-2 S protein,

248 respectively; (G) Bottom view of S protein structure; (H-I) Bottom views of electrostatic

249 potential on surfaces of SARS-CoV and SARS-CoV-2 S protein, respectively. Negatively and

250 positively charged areas are colored in red and blue respectively, with the color scale from -1.0

251 to $1.0 \mathrm{kT} / \mathrm{e}$.

\subsection{Electric Filed Lines}

254 Electric field lines surrounding the two complex structures were calculated. To better visualize 255 the field lines between interfaces, the S protein RBDs are separated from hACE2 RBDs by $10 \AA$ 256 (figure 3). The field lines confirmed that both the SARS-CoV and SARS-CoV-2 S protein RBDs 257 have attractive forces to hACE2 protein. In the analysis of field lines, the density of field lines 
258 indicates the strength of binding force, which means the denser area has the stronger interactions.

259 The electric field lines demonstrate that when hACE2 is away from S protein, all the three S

260 protein monomers provide attractive interactions to the hACE2. This is expected because the $S$

261 protein RBDs are positively charged while the hACE2 is negatively charged, as shown in figure

2622 and movie 3, respectively. When hACE2 binds to S proteins (as shown in figure 1), the hACE2

263 only binds with one S protein RBD, which is in open state. Combining the information from

264 figure 1 and 3, it demonstrates that all the three $S$ protein RBDs generate attractive forces to

265 hACE2. However, when hACE2 gets closer to S protein, one $\mathrm{S}$ protein RBD flips out and binds

266 to the hACE2 tightly, while the other two S protein RBDs stay in closed state. Even though the

267 monomer with flipped-out S protein RBD is the closest to hACE2 and forms most of the salt

268 bridges and hydrogen bonds, the other two monomers also provide dense field lines and show

269 strong attractive interactions between S proteins and hACE2. 


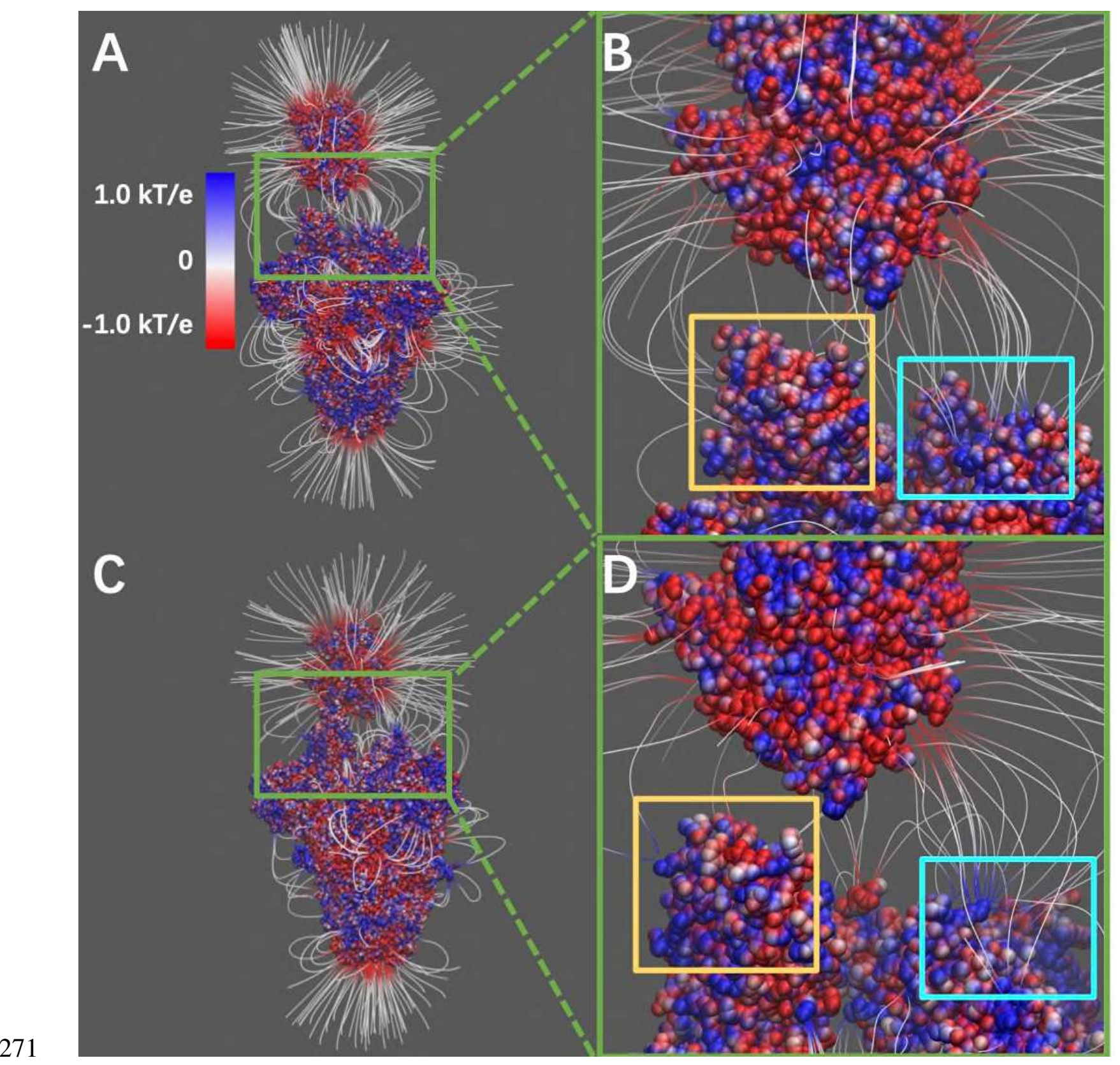

273 Figure 3. Electrostatic filed lines at the interfaces of S protein and hACE2. (A) Electrostatic

274 filed lines between SARS-CoV S protein and hACE2; (B) A closeup view of binding domain

275 between SARS-CoV S protein and hACE2 (C) Electrostatic field lines between SARS-CoV-2 S

276 protein and hACE2; (D) A closeup view of binding domain between SARS-CoV-2 S protein and 277 hACE2. Negatively and positively charged areas are colored in red and blue, respectively. Color 278 scale is -1.0 to $1.0 \mathrm{kT} / \mathrm{e}$. Yellow square areas are the RBD of S proteins at open state to reach the 279 hACE2, cyan square areas are the the RBD of S proteins at closed state. 


\section{4 pH-Dependence of Relative Folding Energies}

282 The folding energy of SARS-CoV and SARS-CoV-2 complexes were calculated using

283 DelPhiPKa at different $\mathrm{pH}$ values ranging from 0 to 14 with an interval of 0.5 (Figure 4). We

284 observed that SARS-CoV and SARS-CoV-2 have the same trend of folding energy with the

285 change of $\mathrm{pH}$ values, which is decreasing from 0 to 6 , then becoming stable from 6 to 9 , and

286 increasing from 10 to 14. Other than the trend, the optimal values locate between 6 to 9 for both

287 of the viruses.

288 Please note that the folding energies in figure 4 are relative values because we set the reference 289 energy to be $0 \mathrm{kcal} / \mathrm{mol}$ when $\mathrm{pH}$ is equal to 0 . We did not calculate the absolute values of 290 folding energies since we focused on the $\mathrm{pH}$ dependency of the folding energies.

\section{Folding energy of S proteins}

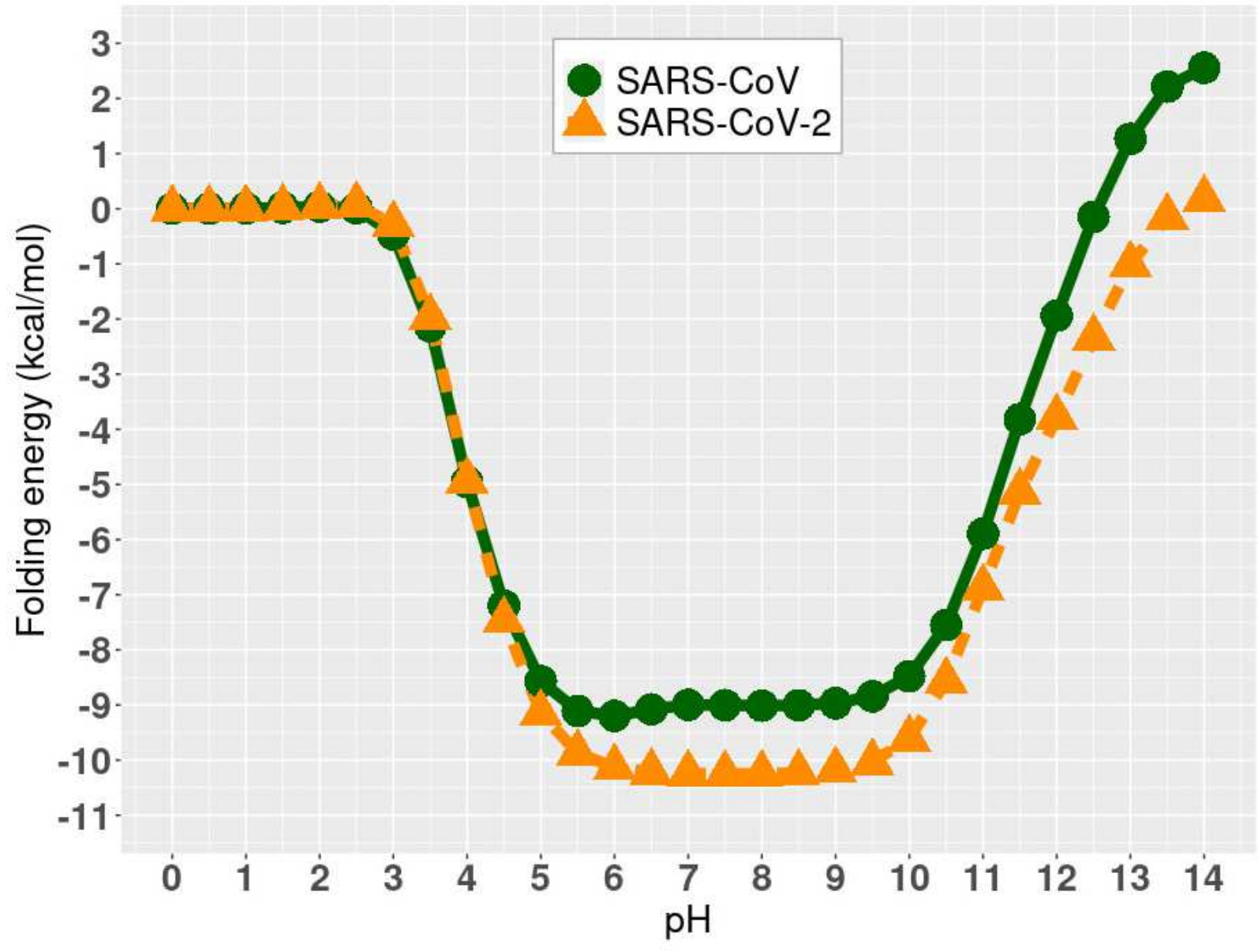

Figure 4. $\mathrm{pH}$-dependence of the relative folding energy of $\mathrm{S}$ protein RBDs of SARS-CoV and 294 SARS-CoV-2. 


\section{5 pH-Dependence of Relative Binding Energies}

297 DelPhiPKa was implemented to calculate the binding energies of two complex structures at different $\mathrm{pH}$ values. The results are presented in figure 5, where we noticed that the binding free energies of both SARS-CoV and SARS-CoV-2 complexes are stable at the $\mathrm{pH}$ values ranging from 7.5 to 10.5, which indicates that both SARS-CoV and SARS-CoV-2 have a slight preference of weakly basic environment. Note that the method implementing DelPhiPKa calculates the relative folding and binding energies rather than absolute energies. The folding/binding energy at $\mathrm{pH} 0$ is set as reference, which is $0 \mathrm{kcal} / \mathrm{mol}$. The relative energy profile is used to study the folding/binding energy dependence on pHs. The absolute binding energies was calculated in later section using MM/PBSA method. Combine the folding and binding energy profiles, it is concluded that the best $\mathrm{pH}$ environment for both the SARS-CoV and SARS-CoV-2 is from $\mathrm{pH} 7.5$ to 9. Please note that the binding energies in figure 5 are relative values because we set the reference energy to be $0 \mathrm{~kJ} / \mathrm{mol}$ when $\mathrm{pH}$ is equal to 0 . We did not calculate the absolute values of binding energies 310 since we focused on the $\mathrm{pH}$ dependency of the binding stability.

\section{pH dependence of binding stability}

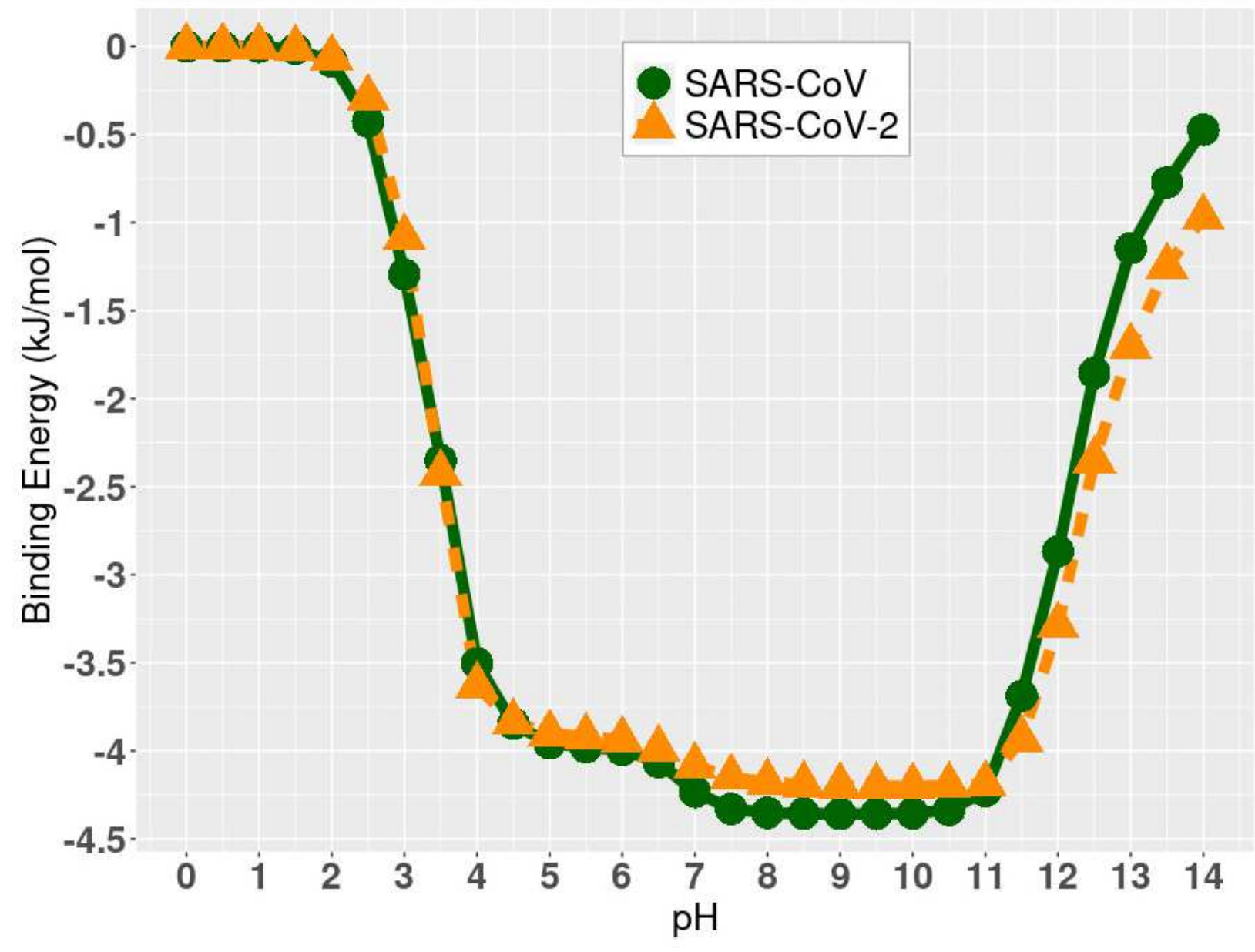

312 Figure 5. The relative binding energies of complexes at different $\mathrm{pH}$ values. 


\section{$314 \quad 3.6 \quad$ Hydrogen Bonds Analysis}

315 To analyze the hydrogen bonds distributions on both S proteins RBDs and hACE2 RBD, we 316 colored the residues forming hydrogen bonds which are over 50\% frequency during the MD 317 simulations in figure 6. It's obvious that the SARS-CoV S protein has more residues involved in

318 the hydrogen bonds which are over 50\%. Accordingly, the hACE also has more residues forming 319 hydrogen bonds (over 50\% frequency) with SARS-CoV S protein.

321 In order to consider the most essential hydrogen bonds, which are the hydrogen bonds with 322 relatively high frequencies, we took $80 \%$ as a cutoff, which means those hydrogen bonds with $32380 \%$ or higher frequency are considered as the relatively more essential ones. By comparing the 324 figure 7A and 7B, SARS-CoV-2 RBD forms one more essential hydrogen bonds than SARS325 CoV RBD when binding to hACE2. The residues involved in forming hydrogen bonds over $50 \%$ 326 frequency were colored with their side chains, in which the residues with over $80 \%$ frequency 327 hydrogen bonds were labeled and highlighted in grey squares (figure 8CF). From the analyses of 328 figure 6-8, it is revealed that SARS-CoV uses more hydrogen bonds to bind with hACE2.

329 However, more high frequency hydrogen bonds are formed in the SARS-CoV-2/hACE2

330 complex. The key residues forming essential hydrogen bonds from SARS-CoV-2 are: ARG-121, 331 TYR103, THR182 and TYR171. Such residues have significant contributions to the binding of 332 SARS-CoV-2 and hACE2. Therefore, these residues have higher potential to be targets for future 333 drug design. 


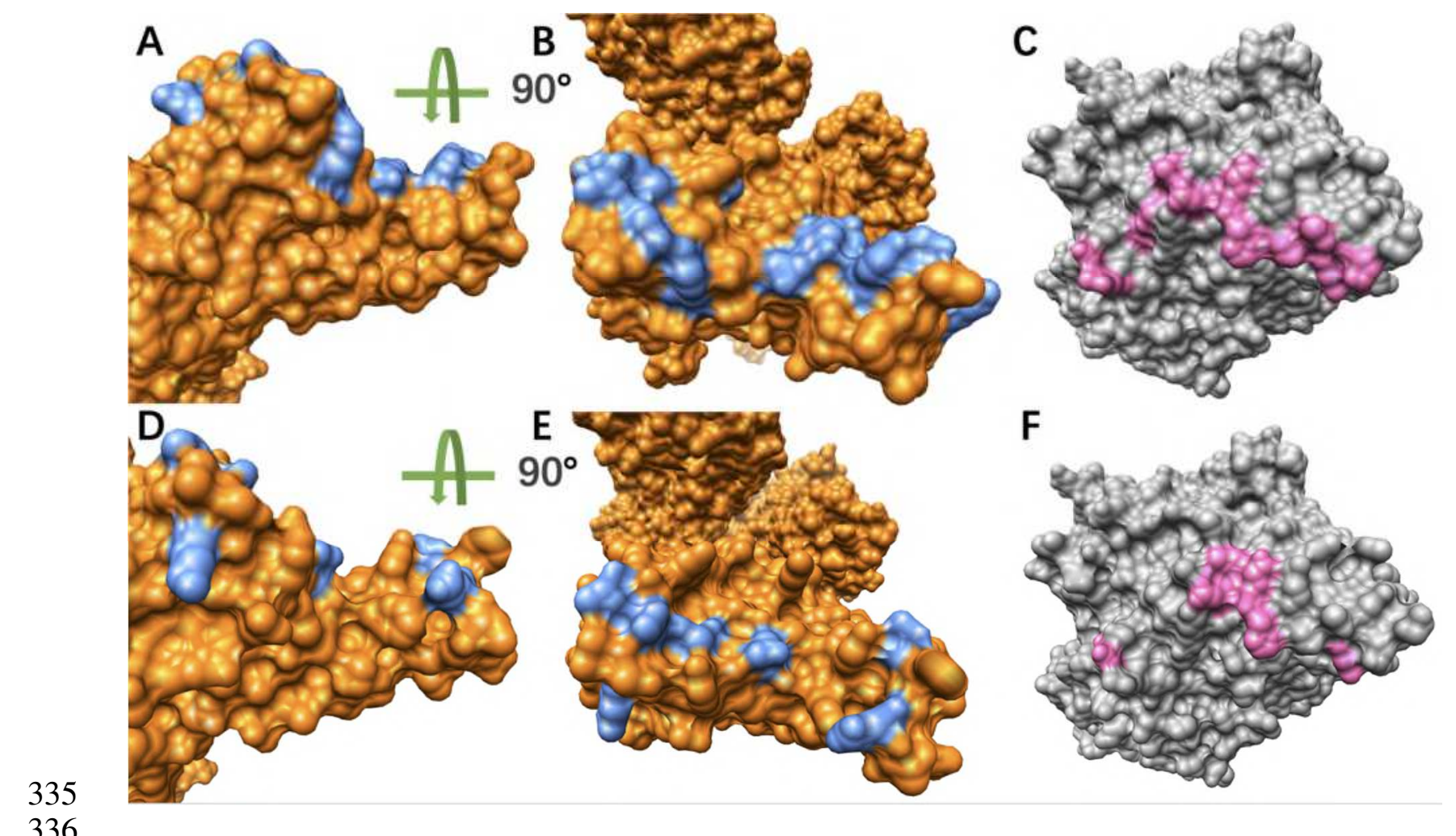

337 Figure 6. Hydrogen bonds distributions at the binding interfaces. (A) Hydrogen bonds

338 distribution (blue) on the interface of SARS-CoV RBD (orange); (B) Turn (A) for 90 degree for

339 the top view, which is the interface that faces hACE2; (C) The hydrogen bonds distribution

340 (pink) at the interface of hACE2 (grey) where SARS-CoV binds; (D) Hydrogen bonds

341 distribution (blue) on the interface of SARS-CoV-2 RBD (orange); (E) Turn (D) for 90 degree

342 for the top view, which is the interface that faces hACE2; (F) The hydrogen bonds distribution

343 (pink) at the interface of hACE2 (grey) where SARS-CoV-2 binds. 

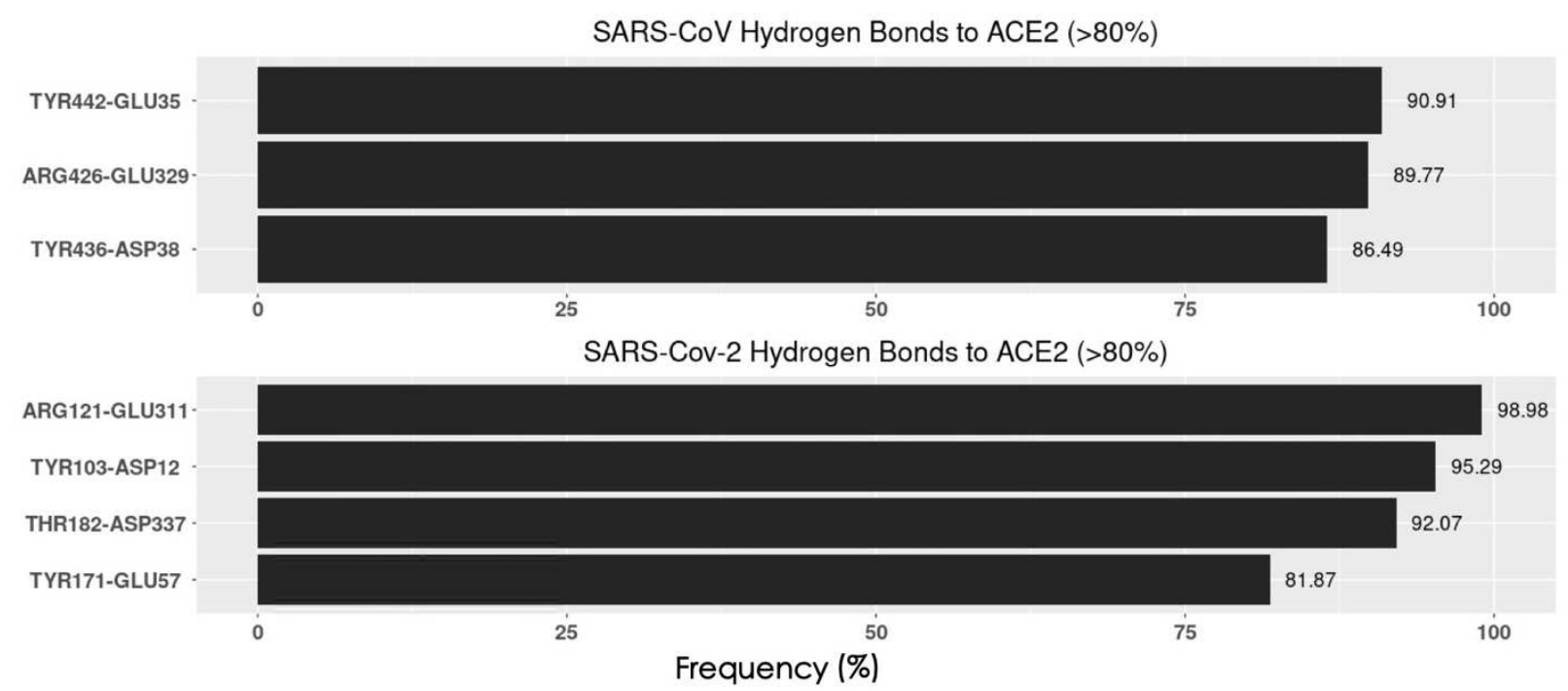

350

Figure 7. Essential Hydrogen bonds at the interfaces between SARS-CoV/SARS-CoV-2 RBDs and hACE2 RBD with the frequency above $80 \%$.

A

Figure 8. Key residues involved in essential hydrogen bonds at the interfaces between SARSCoV/SARS-CoV-2 RBDs and hACE2 RBD with the frequency above 80\%. (A) SARS-CoV S protein single chain binds to hACE2; (B) A closeup view of (A) at the binding interface; (C) 
Labelled key residues that form essential hydrogen bonds (frequency over $80 \%$ ) at the interface; (D) SARS-CoV-2 S protein single chain binds to hACE2; (E) A closeup view of (D) at the binding interface; (F) Labelled key residues that form essential hydrogen bonds (frequency over $80 \%)$ at the interface.

\section{$4 \quad$ Limitation}

362 The limitation for this work is that we used relative folding energy and binding energy to analyze 363 rather than the absolute values. Since our work is focused on the relative stability under the $\mathrm{pH}$ 364 effects, the relative energy calculations do not affect our conclusions.

\section{Conclusion}

In this work, we applied several computational methods, including MD simulations, DelPhi, DelPhiForce and DelPhiPKa to study the electrostatic features of S proteins for SARS-CoV and SARS-CoV-2. From our results, SARS-CoV and SARS-CoV-2 S protein RBDs both have positively charged interfaces, which provides attractive interactions to hACE2 as hACE2 has negatively charged surface.

Also, we revealed the $\mathrm{pH}$-dependence calculations of relative folding energy for SARS-CoV and SARS-CoV-2 S protein RBDs. The best $\mathrm{pH}$ to stabilize SARS-CoV and SARS-CoV-2 S protein RBDs is in the range of 6 to 9 . The study on $\mathrm{pH}$ dependence of binding energies revealed that the complex structures of hACE2 and S proteins of SARS-CoV/ SARS-CoV-2 are stable from $\mathrm{pH}$ 7.5 to 10.5 . Therefore, SARS-CoV and SARS-CoV-2 survive in a similar $\mathrm{pH}$ environment. The pH 7.5 to 9 is the best condition for both SARS-CoV and SARS-CoV-2 to best perform their functions to bind with hACE2.

Besides, based on 100ns MD simulations, we found that for the essential hydrogen bonds (>80\% frequency), SARS-CoV-2 has four pairs while SARS-CoV has three pairs, which indicates the relatively more robust binding strategy of SARS-CoV-2 compared to SARS-CoV. The key residues forming essential hydrogen bonds from SARS-CoV-2 are ARG-121, TYR103, THR182 and TYR171, which are potential drug targets for COVID-19 treatments. By using multiple computational approaches, the findings in this work shed light on the current and future treatments of COVID-19 and other coronaviruses-caused diseases.

\section{Acknowledgement}


This research is funded by Grant SC1GM132043 from National Institutes of Health (NIH); Grant

393 5U54MD007592 from the National Institutes on Minority Health and Health Disparities

394 (NIMHD), a component of the NIH.

\section{References}

1. Cascella, M., Rajnik, M., Cuomo, A., Dulebohn, S.C. and Di Napoli, R., Features, evaluation, and treatment of coronavirus (COVID-19). Statpearls [internet], 2021.

2. Zhou, P., Yang, X.-L., Wang, X.-G., Hu, B., Zhang, L., Zhang, W., Si, H.-R., Zhu, Y., Li, B. and Huang, C.-L., A pneumonia outbreak associated with a new coronavirus of probable bat origin. nature, 2020. 579(7798): p. 270-273.

4. Hadi, A., Werge, M., Kristiansen, K.T., Pedersen, U.G., Karstensen, J.G., Novovic, S. and

3. Van Der Hoek, L., Pyrc, K., Jebbink, M.F., Vermeulen-Oost, W., Berkhout, R.J., Wolthers, K.C., Wertheim-van Dillen, P.M., Kaandorp, J., Spaargaren, J. and Berkhout, B., Identification of a new human coronavirus. Nature medicine, 2004. 10(4): p. 368-373. Gluud, L.L., Coronavirus disease-19 (COVID-19) associated with severe acute pancreatitis: case report on three family members. Pancreatology, 2020. 20(4): p. 665667.

5. Liu, D.X., Liang, J.Q. and Fung, T.S., Human coronavirus-229E,-OC43,-NL63, and-HKU1. Reference Module in Life Sciences, 2020.

6. Loeffelholz, M.J. and Tang, Y.-W., Laboratory diagnosis of emerging human coronavirus infections-the state of the art. Emerging microbes \& infections, 2020. 9(1): p. 747-756.

7. Yang, J., Wang, W., Chen, Z., Lu, S., Yang, F., Bi, Z., Bao, L., Mo, F., Li, X. and Huang, Y., $A$ vaccine targeting the RBD of the S protein of SARS-COV-2 induces protective immunity. Nature, 2020. 586(7830): p. 572-577.

8. Shang, J., Wan, Y., Luo, C., Ye, G., Geng, Q., Auerbach, A. and Li, F., Cell entry mechanisms of SARS-CoV-2. Proceedings of the National Academy of Sciences, 2020. 117(21): p. 11727-11734.

9. Wang, Q., Zhang, Y., Wu, L., Niu, S., Song, C., Zhang, Z., Lu, G., Qiao, C., Hu, Y. and Yuen, K.-Y., Structural and functional basis of SARS-CoV-2 entry by using human ACE2. Cell, 2020.

10. Brielle, E.S., Schneidman-Duhovny, D. and Linial, M., The SARS-CoV-2 exerts a distinctive strategy for interacting with the ACE2 human receptor. Viruses, 2020. 12(5): p. 497.

11. Lan, J., Ge, J., Yu, J., Shan, S., Zhou, H., Fan, S., Zhang, Q., Shi, X., Wang, Q. and Zhang, L., Structure of the SARS-CoV-2 spike receptor-binding domain bound to the ACE2 receptor. Nature, 2020. 581(7807): p. 215-220. 
12. Wang, J., Fast identification of possible drug treatment of coronavirus disease-19 (COVID-19) through computational drug repurposing study. Journal of chemical information and modeling, 2020. 60(6): p. 3277-3286.

13. Li, L., Wang, L. and Alexov, E., On the energy components governing molecular recognition in the framework of continuum approaches. Frontiers in molecular biosciences, 2015. 2: p. 5.

14. Li, L., Guo, D., Huang, Y., Liu, S. and Xiao, Y., ASPDock: protein-protein docking algorithm using atomic solvation parameters model. BMC bioinformatics, 2011. 12(1): p. 1-9.

15. Allen, M.P., Introduction to molecular dynamics simulation. Computational soft matter: from synthetic polymers to proteins, 2004. 23(1): p. 1-28.

16. Liwo, A., He, Y. and Scheraga, H.A., Coarse-grained force field: general folding theory. Physical Chemistry Chemical Physics, 2011. 13(38): p. 16890-16901.

17. Lopez-Hernandez, A.E., Xie, Y., Guo, W. and Li, L., The electrostatic features of dengue virus capsid assembly. Journal of Computational Biophysics and Chemistry, 2021.

18. Guo, W., Xie, Y., Lopez-Hernandez, A.E., Sun, S. and Li, L., Electrostatic features for nucleocapsid proteins of SARS-CoV and SARS-CoV-2. Mathematical Biosciences and Engineering, 2021. 18(3): p. 2372-2383.

19. Peng, Y. and Alexov, E., Computational investigation of proton transfer, $p K$ a shifts and $p$ $H$ - optimum of protein - DNA and protein - RNA complexes. Proteins: Structure, Function, and Bioinformatics, 2017. 85(2): p. 282-295.

20. Wang, L., Witham, S., Zhang, Z., Li, L., Hodsdon, M.E. and Alexov, E., In silico investigation of $\mathrm{pH}$-dependence of prolactin and human growth hormone binding to human prolactin receptor. Communications in computational physics, 2013. 13(1): p. 207.

21. Xian, Y., Karki, C.B., Silva, S.M., Li, L. and Xiao, C., The roles of electrostatic interactions in capsid assembly mechanisms of giant viruses. International journal of molecular sciences, 2019. 20(8): p. 1876.

22. Salas, G.G.S., Hernandez, A.E.L., He, J., Karki, C., Xie, Y., Sun, S., Xian, Y. and Li, L., Using computational approaches to study dengue virus capsid assembly. Computational and Mathematical Biophysics, 2019. 7(1): p. 64-72.

23. Xian, Y., Xie, Y., Silva, S.M., Karki, C., Qiu, W. and Li, L., Structure manipulation tool structureMan: A structure manipulation tool to study large scale biomolecular interactions. Frontiers in molecular biosciences, 2020. 7: p. 476.

24. Karki, C., Xian, Y., Xie, Y., Sun, S., Lopez-Hernandez, A.E., Juarez, B., Wang, J., Sun, J. and Li, L., A computational model of ESAT-6 complex in membrane. Journal of Theoretical and Computational Chemistry, 2020. 19(03): p. 2040002. 
25. Li, C., Li, L., Zhang, J. and Alexov, E., Highly efficient and exact method for parallelization

26. Xie, Y., Du, D., Karki, C.B., Guo, W., Lopez-Hernandez, A.E., Sun, S., Juarez, B.Y., Li, H., Wang, J. and Li, L., Revealing the mechanism of SARS-CoV-2 spike protein binding with ACE2. Computing in Science \& Engineering, 2020. 22(6): p. 21-29.

27. Xie, Y., Karki, C.B., Du, D., Li, H., Wang, J., Sobitan, A., Teng, S., Tang, Q. and Li, L., Spike proteins of SARS-COV and SARS-CoV-2 utilize different mechanisms to bind with human ACE2. Frontiers in molecular biosciences, 2020. 7.

28. Hart, O.E. and Halden, R.U., Computational analysis of SARS-CoV-2/COVID-19 surveillance by wastewater-based epidemiology locally and globally: Feasibility, economy, opportunities and challenges. Science of the Total Environment, 2020. 730: p. 138875.

29. Wu, C., Liu, Y., Yang, Y., Zhang, P., Zhong, W., Wang, Y., Wang, Q., Xu, Y., Li, M. and Li, X., Analysis of therapeutic targets for SARS-CoV-2 and discovery of potential drugs by computational methods. Acta Pharmaceutica Sinica B, 2020. 10(5): p. 766-788.

30. Phillips, J.C., Braun, R., Wang, W., Gumbart, J., Tajkhorshid, E., Villa, E., Chipot, C., Skeel, R.D., Kale, L. and Schulten, K., Scalable molecular dynamics with NAMD. Journal of computational chemistry, 2005. 26(16): p. 1781-1802.

31. Humphrey, W., Dalke, A. and Schulten, K., VMD: visual molecular dynamics. Journal of molecular graphics, 1996. 14(1): p. 33-38.

32. Song, W., Gui, M., Wang, X. and Xiang, Y., Cryo-EM structure of the SARS coronavirus spike glycoprotein in complex with its host cell receptor ACE2. PLoS pathogens, 2018. 14(8): p. e1007236.

33. Juraszek, J., Rutten, L., Blokland, S., Bouchier, P., Voorzaat, R., Ritschel, T., Bakkers, M.J., Renault, L.L. and Langedijk, J.P., Stabilizing the closed SARS-CoV-2 spike trimer. Nature communications, 2021. 12(1): p. 1-8.

34. Webb, B. and Sali, A., Comparative protein structure modeling using MODELLER. Current protocols in bioinformatics, 2016. 54(1): p. 5.6. 1-5.6. 37.

35. Li, L., Li, C., Sarkar, S., Zhang, J., Witham, S., Zhang, Z., Wang, L., Smith, N., Petukh, M. and Alexov, E., DelPhi: a comprehensive suite for DelPhi software and associated resources. BMC biophysics, 2012. 5(1): p. 9.

36. Li, L., Li, C., Zhang, Z. and Alexov, E., On the dielectric "constant" of proteins: smooth dielectric function for macromolecular modeling and its implementation in DelPhi. Journal of chemical theory and computation, 2013. 9(4): p. 2126-2136.

37. Dolinsky, T.J., Nielsen, J.E., McCammon, J.A. and Baker, N.A., PDB2PQR: an automated pipeline for the setup of Poisson-Boltzmann electrostatics calculations. Nucleic acids research, 2004.32(suppl_2): p. W665-W667. 
38. Wang, J., Wolf, R.M., Caldwell, J.W., Kollman, P.A. and Case, D.A., Development and testing of a general amber force field. Journal of computational chemistry, 2004. 25(9): p. 1157-1174.

39. Li, L., Jia, Z., Peng, Y., Chakravorty, A., Sun, L. and Alexov, E., DelPhiForce web server: electrostatic forces and energy calculations and visualization. Bioinformatics, 2017. 33(22): p. 3661-3663.

40. Wang, L., Zhang, M. and Alexov, E., DelPhiPKa web server: predicting pKa of proteins, RNAs and DNAs. Bioinformatics, 2015. 32(4): p. 614-615.

41. Wang, L., Li, L. and Alexov, E., pKa predictions for proteins, RNAs, and DNAs with the Gaussian dielectric function using DelPhi $p K a$. Proteins: Structure, Function, and Bioinformatics, 2015. 83(12): p. 2186-2197.

42. Massova, I. and Kollman, P.A., Combined molecular mechanical and continuum solvent approach (MM-PBSA/GBSA) to predict ligand binding. Perspectives in drug discovery and design, 2000. 18(1): p. 113-135.

43. Lee, J., Cheng, X., Swails, J.M., Yeom, M.S., Eastman, P.K., Lemkul, J.A., Wei, S., Buckner, J., Jeong, J.C. and Qi, Y., CHARMM-GUI input generator for NAMD, GROMACS, AMBER, OpenMM, and CHARMM/OpenMM simulations using the CHARMM36 additive force field. Journal of chemical theory and computation, 2016. 12(1): p. 405-413. 


\section{Supplementary Files}

This is a list of supplementary files associated with this preprint. Click to download.

- S1.jpg

- S2.jpg

- Sl.docx 